\title{
Vorläufige medizinhistorische und pathographische Bemerkungen zu Ulrich Bräker
}

\author{
Von Antoinette Stettler
}

Ulrich Bräker (1735-1798), der «Arme Mann im Toggenburg», braucht nicht lange vorgestellt zu werden. Jedermann erinnert sich, von der «Lebensgeschichte» dieses schreibkundigen Bauern mindestens gehört zu haben, in welcher er von seiner Kindheit, seinen Abenteuern in der preußischen Armee, von seiner Flucht aus der Schlacht bei Lowositz und von seiner Rückkehr in die Heimat erzählt, wo er die Salpetersiederei und schließlich einen Baumwollhandel betrieb. Weniger bekannt ist allerdings, daß er daneben auch Tagebücher für die Jahre 1770-1774 und 1779-1798 hinterlassen hat. Provisorische Abschriften finden sich in der sanktgallischen Kantonsbibliothek Vadiana. In einer demnächst erscheinenden Lebenschronik werden alle Tagebucheinträge regestartig aufgeführt.

Diese Tagebücher, welche Bräker seit dem 35. Altersjahr ziemlich regelmäßig führte, enthalten in anschaulicher Weise mannigfaltige Reflexionen zum politischen Geschehen in der Schweiz und im Ausland sowie zur wirtschaftlich/finanziellen Entwicklung seiner Region Toggenburg, ferner auch Notizen zu Familienproblemen und Gesundheitsdingen. Sie stellen eine Geschichtsquelle ersten Ranges dar*. Neben allem anderen sind sie auch von medizinhistorischem und pathographischem Interesse, zumal Bräker ein ausnehmend gesundheitsbewußter Beobachter war.

Ulrich Bräker, der ständig nur knapp über dem Existenzminimum leben konnte, war nie eigentlich kränklich, aber auch nie ganz gesund. Es verwundert daher nicht, daß er sich einer häufigen Krankheitsprophylaxe durch Purgieren und Aderlaß unterzog, zwar nicht ganz so regelmäßig, wie es vorgeschrieben war, aber dennoch oft genug. Ein gewisses Selbstbewußtsein über seine Fortschrittlichkeit in der Durchführung dieser Praxis spricht aus der folgenden Bemerkung: «Es ist doch gut, daß man sein Blut von sich lassen kann ... Mich wundert, wie von altem Gebrauch her das Aderlassen sei. Ich habe wohl von einem Hippokrat gelesen, der es praktizierte, aber ich weiß nicht eigentlich, um welche Zeit er gelebt ... wir haben ja bei uns noch alte Bauersleut, die in ihrem Leben kein Tropfen Blut gelassen .... ${ }^{1}$

* Siehe dazu: Ulrich Bräker. Die Tagebücher des armen Mannes als Geschichtsquelle. 118. Neujahrsblatt, Historischer Verein des Kt. St. Gallen, 1978. 
Allerdings scheint desungeachtet im Toggenburg der Aderlaß in der Frühlingszeit doch sehr verbreitet gewesen zu sein, eine Tatsache, die auch durch die weite Verbreitung der volkstümlichen Aderlaßkalender nahegelegt wird. «Aderlassen, Blutabzapfen geht jetzt in Schwang. Sonntags auch ich und meine Ehehälfte ...»² ${ }^{2}$.

Es scheint also, daß die Praxis des Aderlasses im Toggenburg nach wie vor in Ansehen stand in einer Zeit, als ihre Nützlichkeit bekanntlich andernorts in den Kreisen der akademisch gebildeten Ärzte seit einiger Zeit in Diskussion, wenn nicht schon in Verruf stand. Wir finden damit in dieser Angelegenheit das Kulturgefälle bestätigt, das auch in andern Gebieten zwischen den geistigen Zentren des umliegenden Europa und der Eidgenossenschaft und in dieser zwischen Zürich und dem Toggenburg, d. h. zwischen Stadt und Land, bestand.

Nicht nur für den Stand der Vorbeugungsmaßnahmen unter seiner Mitbevölkerung interessierte sich Ulrich Bräker. Von 1770 bis 1772 besitzen wir aus seiner Feder auch Jahresübersichten über die im Toggenburg vorkommenden Epidemien, wobei die rote Ruhr und die Grippe am häufigsten geherrscht zu haben scheinen. Doch hören wir auch vom Fleckfieber, vom Ergotismus und von den Windpocken. Die Angaben über Epidemien versieht Bräker oft mit Aufzeichnungen über die Wetterverhältnisse. Zum Teil meldet er auch die Anzahl der Todesfälle; doch es bleibt schwierig, diese Zahlen auszuwerten, weil oft nicht angegeben ist, auf welchen Umkreis sie sich beziehen. 1771 sollen nach Bräker in Wattwil, das zwischen 3500-4000 Einwohnern zählte, 300 an Ruhr und Grippe gestorben sein.

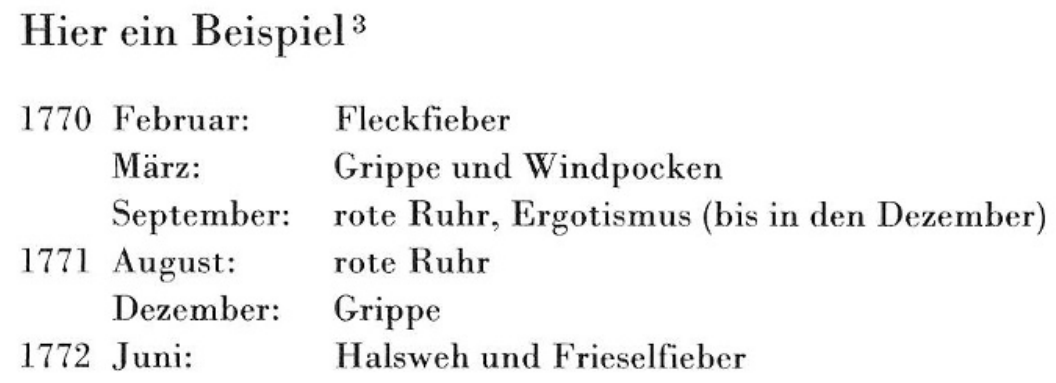

Wenden wir uns nun der Familie von Ulrich Bräker zu, der naturgemäß seine größte Aufmerksamkeit galt, so finden wir sie während der Fleckfieberepidemie vom Februar 1770 «beißig und ausschlägig». ${ }^{4}$ Am schwersten hergenommen wurde seine Familie indessen während der Ruhrepidemie im September 1771, wo innert vier Tagen seine beiden ältesten Kinder 9- und 8jährig starben. 
Die weitaus größte Zahl von Bemerkungen beziehen sich aber auf Bräkers eigenen Gesundheitszustand und seine psychische Verfassung. Für den Leser kristallisieren sich dabei neben interkurrenten Krankheiten katarrhalischer Art, auf die nicht näher eingegangen werden soll, vier größere zusammenhängende Krankheitskomplexe: Da wären erstens ausnehmend häufige und heftige Zahnschmerzen zu nennen.

\begin{abstract}
«Aber heut kommt ein ... Zahnweh hintendrein, ein rasender Schmerz - ich kaute Tabak und probierte allerhand; aber ich ward je länger je wütender, sprang im bloßen Hemd und Kopf ganz wild in die grimmige Kälte hinaus, wälzte den Kopf im Schnee und rieb ihn wacker mit Schnee ... Mein Nachbar ging vorbei und lachte Haut und Lenden voll über mich. - Ich dachte, lach du nur ... Dann wusch ich den Schnee mit kaltem Wasser sauber vom Kopf ... und der Schmerz legte sich. Jetzt hat's mir in alle Glieder gefahren, daß ich halb krank bin und doch viel wöhler.» ${ }^{5}$ «Ein verwünschter Zahn raubte mir alles ... halb konfus und unpaß mußte ich doch eine Reise nach Herisau machen ...» ${ }^{6}$

«Mich hat ein stinkendes Faulfieber und das verhaßte Zahnweh beim Fell, ein Geschwür im Mund machte mir unleidliche Schmerzen. Dachte oft, es wäre mir eine Wohltat, eine Kugel durch den Kopf jagen oder einen Dolch in die Brust stoßen - oh, was man alles denkt, in solch ängstlichen Nächten, wo man vor Schmerz in den wilden Strom hinein springen möchte ... Diese Nacht brach das Geschwür von selbst auf ... Oh, ihr gütigen Mächte, auf einmal wieder wie im Paradies und heute so wohl, obschon ich so blöd und mir selbst vom eignen Gestank zum Ekel bin.» ${ }^{7}$
\end{abstract}

Wir könnten noch einige andere solcher Schilderungen drastischen Schmerzes zitieren, den Ulrich Bräker bisweilen auch mit Schnaps bekämpfte: «hab sie [die Schmerzen] wie schon oft vertrunken ... machte ich es eben wieder». ${ }^{8}$ Im Winter 1783/84 klagt er besonders oft über Zahnschmerzen und vom August bis Oktober 1784 über ein von ihm «Migreinie» genanntes heftiges Kopfweh.

«Unter den rasendsten Kopfschmerzen ging ich vorgestern auf Herisau ... Abends brauchte [ich] das Bad in der Bachstraße - es tat mir gut - gestern aber kam das wütende Migreinie wieder, ... dann mußte ich den Kopf tragen wie ein schalenloses Ei ...» ${ }^{9}$ «Der Wurm im Kopf wühlt noch alle Tage.» ${ }^{10}$ «Eines solchen empfindlichen Schmerzes weiß ich mich nicht zu erinnern, den einzigen Trost hatt' ich dabei, daß es regelmäßig kam, und [ich] die Zeit wußte, wann es wieder abnahm. Mit Sonnenaufgang ging's an ... nahm zu bis gegen Mittag.» ${ }^{11}$

Bisweilen verspürte Bräker durch einen Aderlaß Erleichterung, aber besser half ein Kataplasma aus Eiweiß, Mehl und Branntwein, das er sich auf die Schläfenader legte.

Wir gehen wohl nicht fehl, wenn wir in den rasenden Kopfschmerzen nicht eine Migräne im heute definierten Sinne diagnostizieren, sondern eher 
an eine Arteritis temporalis oder an eine Horton-Neuralgie (Erythroprosopalgie) auf der Basis einer chronischen Kieferhöhleneiterung denken, zumal diese Kopfschmerzattacken weder vorher noch nachher je wieder erwähnt werden. Es ist bemerkenswert, daß Bräker nirgends die Extraktion seiner schlechten Zähne erwähnt, damals immerhin eine gängige Praxis.

Als zweiter Krankheitskomplex fordern Anfälle unsere Aufmerksamkeit, denen Bräker einige Male in seinem Leben unterworfen war:

«Ein Anfall, den ich schon oft hatte, wo sich das Blut empört; erst fangt's mir an in den Händen zu wimseln, dann dringt's mir gegen das Herz, verlegt mir den Atem, daß mir's Angesicht aufschwillt, rot und blau wird.» ${ }^{12}$

«Hatte morgens wieder einen Anfall von einer Blutaufwallung, was mir wie schon oft ... fast den Atem verlegt ... die Lefzen aufschwillt, daß ich im Gesicht blutrot werde und Lippen wie Bratwürste bekomme. Ich muß es glauben, daß es von der Vollblütigkeit herkomme, wie Herr Doktor Mettler sagt, denn nach dem Aderlassen spüre ich nichts mehr davon. Sonst kommt dieser Anfall am ehesten, wenn ich gegen die Diät gesündigt oder etwas Fettes gegessen.» ${ }^{13}$

Wir vermuten bei diesen Anfällen wohl richtig eine Lebensmittelallergie mit Quincke-Ödem im Rachenbereich und Anschwellung der Epiglottis, was die Atemnot erklärt. Unsere These könnte vielleicht auch dadurch gestützt werden, daß der Aderlaß Erleichterung bringt.

Drittens wenden wir uns der Krankheit zu, die zu Bräkers Tode im September 1798 führte. Abgesehen von einer Gonarthrose, über welche er im April 1798 klagt, erkrankt er etwa um die gleiche Zeit:

«Wie ich aber seit einigen Wochen mit einer Heiserkeit behaftet [bin], die mir das Reden schwer macht und oft Halsweh verursacht, als wenn sich eine Entzündung im Schlund ansetzen wollte, mir Stiche bald in den Hals, bald in die Ohren macht, zudem kommt noch der Husten, der mir, bis ich zum Auswurf komme, viel zu schaffen macht, kurz, daß ich von Tag zu Tag immer schwächer werde.» [Ende Mai.] ${ }^{14}$ «Obschon mein Fleisch von den Knochen verschwunden, meine Kräfte mich verlassen, ein zuckender Schmerz sich bald hie bald da in meinem Knochengehäuse äußert ... obschon der Appetit fehlt ... und wenn mir schon der Schlaf nicht immer zu Gebote steht, [sind meine letzten Lebenstage glücklich.]» ${ }^{12}$ [12.Juli.] ${ }^{15}$ «Von innen wirken drückende Beschwerden, anprellender heftiger Schmerz ... wo man keinen Bissen, keinen Schluck Wasser ohne heftige Schmerzen verschlingen kann» [26.Juli.] ${ }^{16}$ «Das Fleisch ist von meinen Knochen verschwunden. Die Kräfte sind dahin, nichts als Reißen in allen Gliedern, Schmerz folgt auf Schmerz, ein unerbittlicher Husten raubt mir mehr als die Hälfte des süßen Schlafes, der immerwährende Schmerz im Hals läßt mich keinen Bissen, kein Glas Wasser ohne heftige Schmerzen verschlingen. Ich stehe kraftlos da wie ein pures Skelett.» [7. August.] ${ }^{17}$

Am 11.September 1798 wurde Ulrich Bräker in Wattwil begraben. 
Angesichts der im Vordergrund stehenden Schluckbeschwerden und der starken Abmagerung darf Bräkers akurat beobachtete Krankengeschichte als sicherlich klassische Schilderung der Symptomatologie eines Larynxkarzinoms gelten. Wenn man noch in Betracht zieht, daß er während Jahrzehnten Tabak rauchte, gewinnt die Diagnose an Wahrscheinlichkeit.

Schließlich verdient als viertes seine psychische Verfassung noch einige Bemerkungen. Wie schon aus den beigefügten Zitaten hervorgeht, war Ulrich Bräker ein ausgezeichneter Beobachter, der sich auch sehr geschickt auszudrücken vermochte. Nicht nur seine Krankheiten weiß er anschaulich zu machen, sondern im Vorbeigehen ist aufgefallen, daß er ein sehr lebhaftes Temperament hatte. Wenn er sich aus Verzweiflung über sein Zahnweh im Schnee wälzt und ihn sein Nachbar auslacht, wünscht er diesem gleich noch ein schlimmeres Stechen an den Hals. Auch sind seine Tagebuchnotizen voll vom Streit mit seiner Ehefrau, die er auch seine Xantippe und sein ihm auferlegtes Kreuz nennt. Gleichfalls häufig sind seine Anspielungen auf Anfechtungen, die er vom weiblichen Geschlecht zu erleiden hat. Außerdem muß er im Anschluß an den berufsmäßigen Marktbesuch ein häufiger Gast im Wirtshaus gewesen sein. $\mathrm{Zu}$ diesem extravertierten Temperament kommt noch eine ausgesprochene Phantasiebegabung, die sich im etwas stilisierten Aufzeichnen von Träumen und Tagträumen kundgibt.

«Mir träumte diese Nacht ..., daß ein Mädchen nachts vor mein Fenster [kam, die Familie] und noch ein Weber waren in der Stube und draußen war's stockfinster. Ich reckte die Hand zum Fenster hinaus, um das vermeinte Mädchen im Scherz beim Kopf zu necken. Plötzlich wurde mir ... ein abenteuerlich garstiges Tier an den Arm geklebt in der Größe einer Kröte, aber platter mit unzählbarer Menge kleiner Beinerchen ... Mir war freilich Angst und Bang, dieses Tier wieder vom Leibe zu bringen ... Ich weiß mich in meinem Leben nicht zu erinnern, daß ich ein solches Tier auch nur unter tausend Kupfern gesehen hätte ... Nun, der Weber ... wollte es mir wegreißen, nahm ein Messer ... und zehrte ... es mit Gewalt von der Haut weg... schlenkerte es auf den Boden ... [wo es sich an den Fuß des jüngsten Kindes heftete]. Aber ich erwachte daran, war noch ziemlich erschrocken über meine Träume und machte allerhand Spekulationen und Auslegungen. Der Wächter rief erst 11 Uhr. Gott, dachte ich, wie nett passen diese Träume zu meinem Leben! Was uns anhängt, das hängt sich auch an unsere Kinder, und das, was wir an uns dulden, möchten wir doch nicht gerne an ihnen sehen. Du, gütiger Engel, bist der Mann, der endlich noch dies Ungeheuer [der Fleischeslust] von meinem Arme reißen wird.» ${ }^{18}$

«... träumte mir, ... daß ich vor Belgrad* stand, ein unübersehbares Lager, aufgeworfene Schanzen und hohe Wälle vor mir sah, sah einen großen breiten Fluß und ganze Scharen Regimenter und Rotmäntel über eine Schiffbrücke marschieren und einen Berg hinauf

\footnotetext{
* Schlacht bei Belgrad, 8. Oktober 1789.
} 
avancieren, ich sah Flammen, Rauch und Dampf von einer großen Stadt aufsteigen, ... und hörte doch nicht sonderlich donnern und knallen ... Ich wollte immer 〈Feuer, Feuer〉 rufen, konnte aber keinen Laut von mir geben. Ich wollte meine Flinte auf die türkischen Mauerlöcher abschießen, aber der Flintenstein gab kein Feuer ... mir war, ich hörte ein dumpfes Heulen, Jammern, Zeter und Mordio Schreiens in der Stadt. Ich sah an einem Ende der Stadt die Mauern zusammenkrachen und ein Gewimmel von Turbanen ... Dies dünkte mich der rechte Zeitpunkt, mit aller Macht hineinzustürmen, wollte eilends laufen ... aber ich konnte nicht von der Stelle kommen, mir war, als wenn mir die Füße gebunden in tiefem Sande staken. Ich ward rasend böse, wollte einen Kameraden senden, konnte aber kein lautes Wort reden ... keiner wollte auf mich achten ... ich sah eine weiße Fahne auf der Mauer flattern, hörte einen Kameraden einem andern ins Ohr flüstern: «es ist vorbei, es ist vorbei, Belgrad ist unser! ... Ich wollte hoch aufspringen und 〈Victoria, Victoria, Belgrad ist vorbei!) rufen, doch es wollte mir nicht klingen, und meine Füße staken im Sand, ich hätte zerplatzen mögen.

Und in diesem Moment muß ich heftige Bewegungen im Bett gemacht haben und ein grauses Gekrächze von mir hören lassen, daß mein Weib davon erwachte, mich stark in die Rippe stieß. 〈W as ist es? Was ist es? sagte sie, 〈Du machst ja ein entsetzliches Gespluder.〉 Nun konnte ich reden. «Belgrad ist vorbei, Belgrad ist vorbei, juhei.> sagte ich und meinte, es sei wirklich wahr. (Ei, was, Belgrad), sagte sie, «was geht uns das an! Laß mich schlafen.»»1 ${ }^{19}$

Im Gegensatz zu seinem offensichtlich extravertierten Temperament stehen sehr häufig Phasen depressiver Verstimmtheit, die sich im Tagebuch in langen Aufzeichnungen schuldhaft-pessimistischen Charakters zeigen. Dazu eines der vielen Beispiele:

«... Ich habe nicht die geringste Empfindung noch einige Freude mehr in mir von einem leiblichen Frühling [obwohl es draußen Frühling ist]. ... Überall bin ich umgeben mit Angst, Kummer, Verdruß und Herzeleid. Meine Vergehungen machen mich verzagt, mein Gewissen ist unruhig, die vergeblichen Wünsche bringen mir Unruhe und machen mich mürrisch. Dazu kommt noch eine unwirsche Krankheit: eine Geißel der Freßbegierde und strenge Arbeit ... Oh, angstvolle Stunden, wenn alles Verdrießlichkeiten dieses Lebens zusammenkommen! Ich hätte bald mit Hiob den Tag meiner Geburt gescholten oder mit Jonas mir den Tod gewünscht ...» ${ }^{20}$

Mit Hilfe pietistischer Religionsbetrachtung und -übung, aber auch mit Alkohol und Laudanum sucht Bräker über diese Verstimmtheiten wegzukommen, wobei dann ab und zu auch leicht manisch-euphorische Tagebucheintragungen folgen.

Einem Mann von Bräkers Begabung und extravertiertem Temperament mußte der Toggenburger Rahmen, und darin seine sehr niedrige Herkunft als ein ungeheures Hindernis zur Selbstentfaltung erschienen sein. Wenn er seine Herkunft durch Autodidaktik wettzumachen suchte, fand er in seiner näheren Umgebung außer in der «Toggenburgischen Moralischen Gesell- 
schaft» weder viel Verständnis noch Unterstützung. Daß er in einer mehrheitlich feindselig eingestellten Umgebung vereinsamte, wo er nur durch geistigen Ehrgeiz, keineswegs durch wirtschaftlichen Erfolg auffiel, verwundert nicht. So sind denn seine depressiven Verstimmungen wohl eher den äußeren Umständen zuzuschreiben, somit reaktiver Natur.

Es ist wohl möglich, daß wir dieser Vereinsamung die Tagebücher verdanken, die uns einen einzigartigen Einblick in ein Leben im 18. Jahrhundert geben. Doch ist andererseits nicht zu vergessen, daß durch das ganze 18. Jahrhundert, vor allem auch in pietistischen Kreisen, die Introspektion und somit das Tagebuchführen gepflegt wurde ${ }^{21}$. Damit können wir Bräkers Tagebücher dieser Literaturgattung zurechnen, deren berühmtestes Beispiel wir in Jean-Jacques Rousseaus «Confessions» finden.

\section{Anmerkungen}

(Umschreibung in zeitgemäße Orthographie vom Autor.) Die Zitate aus Ulrich Bräkers Tagebüchern stammen von den Jahrgängen:

${ }^{1} 1783 / 169$.

${ }^{2} 1785 / 353$.

${ }^{3}$ Übersichten im Anhang zu den Tagebüchern 1770-1772.

${ }^{4} 1770 / 336$.

${ }^{5} 1780 / 4-5$.

${ }^{6} 1780 / 29$.

' $1780 / 34-35$.

${ }^{8} 1783 / 226$.

${ }^{9} 1784 / 315$.

${ }^{10} 1784 / 316$.

${ }^{11} 1784 / 337$.

${ }^{12} 1781 / 166$.

$131783 / 168$.

${ }^{14} 1798 / 89$.

$151798 / 111$.

${ }^{16} 1798 / 121$.

$171798 / 125$.

${ }^{18} 1783 / 238-239$.

19 1789/194-195.

${ }^{20} 1772 / 182$.

${ }^{21}$ Siehe dazu: Christa Habrich, Pathographische und ätiologische Versuche medizinischer Laien, in: Heilberufe und Kranke im 17. und 18. Jahrhundert. Münster 1982. 


\section{Zusammenfassung}

Ulrich Bräker (1735-1798), der «Arme Mann im Toggenburg», schrieb nicht nur seine Lebensgeschichte, sondern führte auch Tagebücher für die Jahre 1770-74 und 1779-98. Diese enthalten eine Fülle von Angaben über den Gesundheitszustand seiner eigenen Person, seiner Familie und seiner Toggenburger Gemeinde. Die vorliegende Arbeit bringt eine erste Übersicht über das in den Tagebüchern vorhandene Material und ist als Anregung zu weiteren medizinhistorischen und pathographischen Untersuchungen gedacht.

\section{Summary}

Ulrich Bräker (1735-1798), the well-known autodidactic peasant, not only wrote the history of his life, but also kept a diary during the years 1770-74 and 1779-98. This diary contains amongst other a great amount of observations pertaining to his state of health as well as that of his family and community in Toggenburg (Switzerland). The above article is the first endeavour to grasp this wealth of information and is intended as an incentive for further investigations.

Dr. med. Antoinette Stettler

Kirchenfeldstraße 45

CH-3005 Bern 